\title{
Preface to the Special Issue: Creativity and Innovations on the Path to Fusion Energy
}

\author{
Vincent Chan ${ }^{1} \cdot$ Minh Quang $\operatorname{Tran}^{2} \cdot$ Glen Wurden ${ }^{3}$
}

Published online: 30 August 2018

(C) Springer Science+Business Media, LLC, part of Springer Nature 2018

The path to fusion energy is sprinkled with numerous innovative ideas. The goal of this special issue is to offer a public forum for developers of all concepts to present articles of an overview nature to bring to the fusion community novel fundamental ideas, status of technological development and path to realization of fusion energy as well as the necessary technology (such as the one necessary to ensure Tritium self-sufficiency). The special issue is a great opportunity to showcase the diversity and creativity in the field of fusion research worldwide. By collecting a broad spectrum of fusion approaches, the required associate technology and making them available in a single special issue, it could stimulate further interests from the scientific public and serve as a valuable reference for researchers in fusion.

The special issue starts with an article "On the use of high magnetic fields in reactor grade tokamaks" by Hartmut Zohm. This paper lays out key arguments that by advancing the superconducting magnet technology beyond existing capability, it could open up multiple pathways to a $\mathrm{GW}_{\mathrm{e}}$ level high field tokamak reactor. The options include a pulsed reactor as a follow-on to ITER. By incorporating a more recent innovative idea called the hybrid scenario, the EU-Demo could extend the ITER design close to a steadystate reactor with potential for higher plasma performance and bootstrap current to reduce the external current drive requirement. Fully non-inductive steady-state operation is possible at high magnetic field by lowering the plasma current to increase the bootstrap current fraction. Both the China Fusion Engineering Test Reactor and the Japan Demo belong to this category. Finally, if advanced physics

Vincent Chan

chanv@fusion.gat.com

1 General Atomics, San Diego, USA

2 Ecole Polytechnique Fédérale de Lausanne, Lausanne, Switzerland

3 Los Alamos National Laboratory, Los Alamos, USA performance could be realized as recent experimental innovations from DIII-D, AUG and other tokamaks suggest, a compact high field tokamak reactor can be realized. Some of the technology challenges not discussed in this paper are elaborated in other papers in this special issue.

ITER is by far the largest fusion science and engineering experiment being designed and constructed to date. The paper by David Campbell, et al., "Innovations in technology and science R\&D for ITER" provides an exhaustive review of the experience and challenges during the course of the design. It gives special prominence to some key ground-breaking $R \& D$ events, including development of high performance $\mathrm{Nb}_{3} \mathrm{Sn}$ superconducting strand in order to construct the largest superconducting magnets, fabrication of high heat flux plasma facing components, controlling and mitigating plasma disruptions, and exploiting new fusion nuclear technologies such as remote maintenance and tritium test blankets for fusion reactor deployments. Besides providing a viable path to fusion demo, the ITER experience is invaluable for the design of all future tokamak reactors.

The Advanced Tokamak (AT) concept provides one of the most promising approaches to meet the challenge of sustained fusion performance. The essence of the approach is to replace inductive current with a combination of 'bootstrap' current and auxiliary non-inductive current drive. Using strong plasma shaping and internal profile control, AT can lead to higher plasma performance, which opens the possibility of a compact tokamak power plant. For this concept to be considered seriously, a strong scientific database is required from existing experiments. The paper by Richard Buttery, et al., "DIII-D research to prepare for steady-state Advanced Tokamak power plants" provides a state-of-the-art overview of AT physics development. The article focuses on DIII-D progress but also provides extensive references of results from other experiments. The authors lucidly explain the development of plasma shape and profile control, which leads to multiple AT operating scenarios. On-going research to address the 
compatibility between core and boundary is described, and future research including upgrades required to establish a firm AT foundation is outlined.

Worldwide, many ITER Parties are developing the step after ITER, often called DEMO. The Chinese fusion program has developed a road map, in which the China Fusion Engineering Testing Reactor (CFETR) plays a key role. The engineering design phase of CFETR was approved by the Chinese government and started in December 2017. CFETR will be developed in two stages: the first stage will explore steady state operation and Tritium self-sufficiency (cf. Paper by Xiaolin Wang, et al., in this issue) albeit at low fusion power $(<200 \mathrm{MW})$. The second stage aims towards a much larger fusion power $(1 \mathrm{GW})$ with requirements and operating space close to DEMO. The physics basis and engineering challenges for CFETR are described in the paper "Present state of Chinese magnetic fusion development and future plan" by Jiangang Li and Yuanxi Wan. To prove Tritium self-sufficiency before proceeding to DEMO, a Tritium fuel processing plant has to be part of CFETR. For this purpose, CFETR has developed a full concept for the Tritium plant, which is described in a companion paper "Current progress of Tritium fuel cycle technology for CFETR" by Xiaolin Wang.

Japan has in place for many years a vigorous DEMO program. Being a key partner with EU in planning the next step beyond ITER, a new superconducting tokamak JT60SA will be commissioned soon to support ITER operation and develop the basis for DEMO. The paper "Review of strategy toward DEMO in Japan and required innovations" by Kunihiko Okano is a discussion of the proposed strategy. It is also recognized (as in many other DEMO approaches) that the construction of DEMO requires innovations in many engineering fields, which are presented in the paper. While many Demonstration Reactor (DEMO) designs are based on the tokamak concept since the leverage of experience from ITER is then straightforward, the stellarator concept is being developed through investments in many large experiments because of its inherently steady-state nature. The paper "Conceptual design of the helical fusion reactor FFHR-d1C with configuration optimization and engineering feasibility of three dimensional structures" by Nagato Yanagi, et al., presents novel optimization of FFHR-d1A based on LHD results. Technical ideas are described for the 3D superconducting coils and the divertor, two of the most challenging and innovative areas in the design.

Three fusion ideas that are in proof-of-concept stage are featured in this special issue. They highlight many innovations that sustain "alternate" fusion pathways as a vibrant and essential component of fusion energy development.
"Encouraging results and new ideas for fusion in linear traps" by Peter Bagryansky, et al., summarizes results from the last 10 years in two classes of linear magnetic mirror machines: epitomized by the GOL-3 multiple mirror, and the GDT gas dynamic trap. The manuscript explains both the benefits and weaknesses of the linear mirror machine approach, and how pathways to overcoming these weaknesses have been systematically identified, and in several cases tested. Even at today's level of development, the gas dynamic trap can be a successful (low-Q) volumetric fusion neutron source. For higher performance as a fusion reactor candidate, the paper describes several modifications that may lead to improved high-beta confinement, and which require next generation open trap mirror test facilities.

"Magnetized Plasma Target for Plasma-Jet-Driven Magneto-inertial Fusion" by Hsu and Langendorf, LANL is a forward-looking design scenario paper that describes modelling for plasma jet formation of a high beta "tangled open-field line" plasma target, followed by subsequent plasma jet driven spherical compression, for magneto-inertial fusion (MIF) experiments. For MIF, one is concerned both with forming a clean plasma target that has a lifetime well matched to the imploding liner characteristics, and with having a possible rep-rated driver system. Of particular interest is a concept known as plasma jet magnetoinertial fusion (PJMIF). The paper uses both analytic estimates and the 1D HELIOS numerical code to identify potential areas of the MIF design space, of interest to a future near term PJMIF hypersonic jet compression experiment.

"Magnetized Target Fusion with a spherical tokamak" by Laberge, General Fusion is a representative paper on fusion R\&D in the private sector. This design paper looks at the potential benefits of compressing a spherical tokamak to $70 \mathrm{~T}$ toroidal magnetic field! By taking a speculative step in mixing and matching plasma parameters from existing plasma devices, it sets up a pre-compression design point. Then it postulates liquid metal air-driven pistons, with highly efficient energy recovery, to arrive at a low gain fusion power plant with $40 \mathrm{MW}$ net output power. The author recognizes there are many elements which will require further study, especially for example, the need for including radiation losses during compression, as well as consideration of plasma stability during the compression to high beta, while avoiding disruptions.

Guest Editors:

Vincent Chan

Minh Quang Tran

Glen Wurden 